\title{
The Italian national surgical site infection surveillance programme and its positive impact, 2009 to 2011
}

M Marchi ${ }^{1,2}$, A Pan (apan@regione.emilia-romagna.it) ${ }^{1,2,3}$, C Gagliotti ${ }^{1}$, F Morsillo ${ }^{1}$, M Parenti $^{1}$, D Resi ${ }^{1,4}$, M L Moro $^{1}$,

the Sorveglianza Nazionale Infezioni in Chirurgia (SNICh) Study Group ${ }^{5}$

1. Area Rischio Infettivo, Agenzia Sanitaria e Sociale Regionale dell'Emilia-Romagna (Infectious risk area, Health and Social Regional Agency of Emilia-Romagna), Bologna, Italy

2. These authors contributed equally to this work

3. Infectious and Tropical Disease Unit, Istituti Ospitalieri di Cremona, Cremona, Italy

4. Dipartimento di Sanità Pubblica, Azienda Unitaria Sanitaria Locale (Department of Public Health, Local Health Agency), Ravenna, Italy

5. The members of the group are listed at the end of the article

Citation style for this article:

Marchi M, Pan A, Gagliotti C, Morsillo F, Parenti M, Resi D, Moro ML, the Sorveglianza Nazionale Infezioni in Chirurgia (SNICh) Study Group. The Italian national surgical site infection surveillance programme and its positive impact, 2009 to 2011. Euro Surveill. 2014;19(21):pii=20815. Available online: http://www.

eurosurveillance.org/ViewArticle.aspx?Articleld=20815

Article submitted on 03 April 2013 / published on 29 May 2014

Programmes surveying surgical site infection (SSI) have been implemented throughout the world and are associated with a reduction in SSI rates. We report data on non-prosthetic surgery from the Italian SSI surveillance programme for the period 2009 to 2011. Participation in the programme was voluntary. We evaluated the occurrence of SSI, based on protocols from the European Centre for Disease Prevention and Control, within 30 days of surgery. Demographic data, risk factors, type of surgery and presence of SSI were recorded. The National Coordinating Centre analysed the pooled data. On 355 surgical wards 60,460 operations were recorded, with the number of surveyed intervention doubling over the study period. SSI was observed in 1,628 cases $(2,6 \%)$ and $60 \%$ of SSI were diagnosed through 30-days post discharge surveillance. Operations performed in hospitals with at least two years of surveillance showed a $29 \%$ lower risk of SSI. Longer intervention duration, American Society of Anesthesiologists' (ASA) score of at least three, and pre-surgery hospital stay of at least two days were associated with increased risk of SSI, while videoscopic procedures had reduced SSI rates. Implementation of a national surveillance programme was helpful in reducing SSI rates and should be prioritised in all healthcare systems.

\section{Introduction}

Surgical site infections (SSI) represent one of the main complications in patients undergoing surgery, with major implications in terms of morbidity, including additional surgical procedures or transfer to an intensive care unit (ICU), mortality, longer duration of hospital stay, and financial burden [1]. A considerable proportion of SSI could be avoided through the implementation of adequate preventive strategies. Thus SSI incidence has been recommended by the European Council and proposed as an indicator of healthcare quality in the context of clinical governance and performance monitoring, and is therefore a target of many healthcare systems [2-4].

Over the past four decades, national and international SSI surveillance systems have been implemented, aimed at gathering data on SSI and building programmes to reduce their incidence [5-7]. Although data from different hospitals may vary significantly, due to factors such as hospital and patient characteristics, benchmarking SSI incidence between hospitals and over time may allow identification of areas for targeted intervention and may help to better allocate resources. [8] In addition to documenting a relevant part of the healthcare system, surveillance itself, even without any specific intervention, has been associated with a reduction in SSI incidence, another reason to recommend implementation of national surveillance systems $[5,8,9]$.

The Italian Centre for Disease Control and Prevention (Centro per il Controllo e la Prevenzione delle Malattie; CCM) funded, in 2005, the implementation of a national surveillance system for SSI (Sistema Nazionale Sorveglianza Infezioni del Sito Chirurgico; SNICh), with the aim to facilitate comparisons within and between hospitals and to participate to the European surveillance programme, coordinated by the European Centre for Disease Prevention and Control (ECDC). The objective of this study was to describe the main characteristics of the SNICh programme, and in particular to report its impact on SSI rates for the period from 2009 to 2011.

\section{Methods}

Settings and background

The study was performed within the national surveillance system, coordinated by the Regional Health Agency of the Emilia-Romagna Region (Agenzia 
Sanitaria e Sociale Regionale Emilia-Romagna; ASSR) and funded by CCM. ASSR acted as the National Coordinating Centre. Participation in the SNICh programme is voluntary. Any single hospital ward, hospital, or regional network may participate.

\section{Data collection}

The procedures undergoing surveillance are those reported in the National Protocol for SSI surveillance [10]. For this study, procedures involving implants of prosthetic material were not considered due to the very different length of post-intervention follow-up that is required (one year vs one month) and because data regarding the one-year follow-up were not yet available at the moment of the analysis. Furthermore, intervention categories that did not reach 100 operations in the considered time frame were excluded. Information on SSIs were recorded by clinicians and/or nurses during post-operatory contacts with patients. This analysis included surgical procedures surveyed between 2009 and 2011 from all participating surgical wards.

Data on surgical operations are recorded, by law, for every procedure, in the hospitals' operation registries. Thus information on surgical unit, date of operation, procedure ICD-9CM codes, wound contamination class, American Society of Anaesthesiology (ASA) score, duration of intervention, whether the procedure was urgent and whether it was performed using an endoscopic/laparoscopic approach, was retrieved by hospital staff from the hospital operation registry. Demographic characteristics of patients (sex and age) were recorded in the discharge form (Scheda di Dimissione Ospedaliera; SDO) of every patient staying in the hospital. Dates of admission and discharge and the hospital identification code were also retrieved from the SDO.

Information on post-discharge contact were retrieved from three different sources, depending on the type of contact: (i) if the patient was readmitted to the hospital within the follow-up time frame, data were available via SDO; (ii) whenever the patient accessed the hospital for post-discharge visits, data were available via the regional ambulatory activity database (ASA); (iii) in case the information regarding the intervention followup was obtained by phone or on returning the post-discharge letter, a special form was filled in. If more than one type of information was available, the latest date within 30 days from the intervention was considered as the 'date of last information' and therefore recorded in the database. Data regarding the number of surgical procedures performed in Italy were retrieved from the Ministry of Health database [11].

Data were fed back to all participating centres in the form of written reports in three different formats: a pdf file for the national report published on the SNICh site, an html dashboard for regional reports and another html report for single-hospital reports. The national report is published once a year. The regional and single-hospital analyses are sent via email to all participating centres and to regional contact points once a year or on demand.

\section{Definitions}

The main outcome variable was the occurrence of an SSI within 30 days of the operation. SSIs were further classified as superficial, deep incisional, or organ/ space. The definitions used for recording SSIs and classifying them for severity were those given by ECDC in the "hospital acquired infection surgical site infection' (HAISSI) protocol [12].

Wound Classification, ASA score, and duration of intervention were used to calculate the SSI risk index [13]. Definitions by ECDC were used for this group of variables. The cut-off values for the duration of operative procedures categories, needed for the calculation of SSI risk index, were taken from the protocol of the National Health Surveillance Network (NHSN) [14]. ICD${ }_{9} \mathrm{CM}$ procedures codes were grouped into operative procedure categories according to the NHSN.

A variable indicating how long a hospital had been performing SSI surveillance was calculated for every procedure in the database as the difference between the surgery date and the date when the hospital started performing surveillance. When a hospital interrupted the surveillance for a full quarter, a new starting date was used for later procedures. The variable was then recoded as a binary one, indicating whether the hospital had been surveying for more than two years at the time of one particular surgery.

\section{Statistical analysis}

Continuous variables, such as age and duration of operation were recoded as categorical variables. Duration was recoded as a binary variable indicating whether the procedure lasted longer than the NHSN 75th percentile for that particular category (as it is done when calculating the SSI risk index.) Age was also recoded as a binary variable, by dividing patients into those younger than 65 years and those 65 years and older.

Statistical significance for univariate odds ratios was assessed with Fisher's exact test. Multivariate analysis was performed using generalised mixed models, in order to account for the correlation of episodes within hospitals and operative procedures. The model presented is a multilevel model with random intercepts and the outcome variable following a binomial distribution with a logit link.

The hospital and the operative procedure category were treated as random effects. Wound classification, ASA score, duration of operation, technique used (classical vs laparoscopic/endoscopic) and urgency of operation were treated as fixed effects, as were potential confounders such as age and sex. Finally, the variable indicating whether the hospital in which the operation 
Main characteristics of the operations recorded in the SNICh programme, Italy, 2009-2011 ( $\mathrm{n}=60,460)$

\begin{tabular}{|c|c|c|}
\hline & $\begin{array}{c}\text { Operations } \\
\text { n (\%) }\end{array}$ & $\begin{array}{c}\text { Infections } \\
\text { n (rate per } 100 \\
\text { procedures) }\end{array}$ \\
\hline \multicolumn{3}{|l|}{ Duration of operation } \\
\hline Under $75^{\text {th }}$ percentile & $48,438(80)$ & $1,108(2.3)$ \\
\hline Over $75^{\text {th }}$ percentile & $12,022(20)$ & $520(4 \cdot 3)$ \\
\hline \multicolumn{3}{|l|}{ ASA score } \\
\hline 1 & $18,085(30)$ & $285(1.6)$ \\
\hline 2 & $26,019(43)$ & $712(2.7)$ \\
\hline 3 & $9,410(16)$ & $422(4 \cdot 5)$ \\
\hline 4 & $1,804(3)$ & $116(6.4)$ \\
\hline 5 & $152(0)$ & $9(5.9)$ \\
\hline NA & $4,990(8)$ & $84(1.7)$ \\
\hline \multicolumn{3}{|l|}{ Wound class $^{\text {a }}$} \\
\hline 1 & $29,055(49)$ & $478(1.6)$ \\
\hline II & $23,844(40)$ & $673(2.8)$ \\
\hline III & $4,947(8)$ & $318(6.4)$ \\
\hline IV & $1,488(3)$ & $152(10.2)$ \\
\hline \multicolumn{3}{|l|}{ Technique of operation ${ }^{a}$} \\
\hline Classic & $46,911(79)$ & $1,414(3.0)$ \\
\hline Videoscopic & $12,125(21)$ & $211(1.7)$ \\
\hline \multicolumn{3}{|c|}{ Hospital stay before operationa } \\
\hline$<2$ days & $28,499(47)$ & $485(1.7)$ \\
\hline$\geq 2$ days & $31,917(53)$ & $1,141(3.6)$ \\
\hline \multicolumn{3}{|l|}{ Sex } \\
\hline Male & $20,298(34)$ & $668(3.3)$ \\
\hline Female & $40,162(66)$ & $960(2.4)$ \\
\hline \multicolumn{3}{|l|}{ Age $^{a}$} \\
\hline $0-1$ & 399 (1) & $7(1.8)$ \\
\hline $2-5$ & $470(1)$ & $6(1.3)$ \\
\hline $6-15$ & $955(2)$ & $23(2.4)$ \\
\hline $16-45$ & $21,778(36)$ & $376(1.7)$ \\
\hline $46-65$ & $16,262(27)$ & $461(2.8)$ \\
\hline $66-85$ & $18,533(31)$ & $690(3.7)$ \\
\hline$\geq 85$ & $1,955(3)$ & $65(3.3)$ \\
\hline \multicolumn{3}{|l|}{ Urgent operation $^{\mathrm{a}}$} \\
\hline No & $45,044(75)$ & $1,174(2.6)$ \\
\hline Yes & $15,006(25)$ & $452(3.0)$ \\
\hline \multicolumn{3}{|c|}{ Operative procedure category ${ }^{b}$} \\
\hline Caesarean section & $12,970(21)$ & $222(1.7)$ \\
\hline Cholecystectomy & $9,653(16)$ & $162(1.7)$ \\
\hline Breast surgery & $8,724(14)$ & $156(1.8)$ \\
\hline Colon surgery & $6,130(10)$ & $508(8.3)$ \\
\hline Herniorrhaphy & $4,172(7)$ & $50(1.2)$ \\
\hline Open reduction of fracture & $2,365(4)$ & $14(0.6)$ \\
\hline Appendectomy & $1,957(3)$ & $51(2.6)$ \\
\hline Prostatectomy & $1,558(3)$ & $49(3.1)$ \\
\hline Rectal surgery & $1,412(2)$ & $126(8.9)$ \\
\hline Laminectomy & $1,407(2)$ & $5(0.4)$ \\
\hline Thoracic surgery & $1,010(2)$ & $11(1.1)$ \\
\hline
\end{tabular}

ASA: American Society of Anaesthesiology; SNICh: Sistema Nazionale Sorveglianza Infezioni del Sito Chirurgico.

a Data are missing in these categories. Percentages are calculated on the available data: wound class $(n=59,334)$, operation technique $(n=59,036)$, hospital stay before operation

$(n=60,416)$, age $(n=60,352)$, urgent operation $(n=60,050)$.

${ }^{b}$ Only operation categories with $\geq 1,000$ procedures are reported. was performed had been continuously submitting data to the SNICh system for more than two years (at the time of operation) was also treated as a fixed effect.

Different model specifications were evaluated. Continuous variables were tested without being recoded as categorical ones, different groupings were tried for categorical ordinal variables, components of the SSI risk index were replaced by the index itself and random slopes were added to random effects in the hypothesis that operations characteristics have different effects on the outcome depending on the operative procedure category. The significance of the random effects was assessed by comparing the log-likelihoods of models. Alternative models either gave worse results than the one presented here (according to Akaike's and Bayesian information criteria) or introduced complexity without providing a significant improvement.

The model presented for the main outcome variable was also applied to a second end point, in-hospital detected severe infections (either deep incisional or organ/space).

Data were analysed with the statistical software $R$ [15]. The R package Ime4 [16] was used for the multilevel modelling and the R package exactci [17] was used for calculating confidence intervals.

\section{Results}

The SNICh system collected data on 83,127 operations from 2009 to 2011, and the final number of operations considered for the study was 60,460 . For 54,240 of these $(89.7 \%)$ there was no missing information.

The surveyed operations increased from 14,616 in 2009 to 28,739 in 2011 (+96\%). The top 10 interventions surveyed in 2011 represented $3 \%$ of the interventions performed at the national level, varying between 2.3\% for appendectomy and 5.9\% for breast surgery. A total of 355 wards, from 12 of the 20 Italian regions, contributed an average of 170 records (median: 64; interquartile range (IQR): 23-147.) Two thirds of the patients were females, and the combined average age was 53 years. Female patients were on average significantly younger (51 vs 59 years; t-test: 46.35; p<0.001), but the difference was entirely due to Caesarean section operations (after removing Caesarean sections, both sexes averaged at 59 years of age.)

Pre-operatory hospital stay lasted for a median of two days (IQR: 1-3), while post-operatory stay lasted for a median of three days (IQR: 1-6). Thirty percent of the patients were operated in a hospital which had been continuously reporting data to SNICh for more than two years (at the time of operation.) Distributions of characteristics of the operations are reported in Table 1.

An SSI was reported for 1,628 operations (2.6\%); 544 infections were either deep incisional or organ/space: 
Variables associated with surgical site infections: univariate and multivariate odds ratios, Italy, 2009-2011 (n=1,628)

\begin{tabular}{|c|c|c|c|c|c|c|}
\hline & \multicolumn{3}{|c|}{ Univariate analysis } & \multicolumn{3}{|c|}{ Multivariate analysis ${ }^{a}$} \\
\hline & OR & $95 \% \mathrm{Cl}$ & $\mathrm{p}$ value & OR & $95 \% \mathrm{Cl}$ & $\mathrm{p}$ value \\
\hline \multicolumn{7}{|l|}{ Duration of operation ${ }^{b}$} \\
\hline Under 75 th percentile & 1 & - & - & 1 & - & - \\
\hline Over 75th percentile & 1.93 & $1.74-2.15$ & $<0.001$ & 1.52 & $1.32-1.74$ & $<0.001$ \\
\hline \multicolumn{7}{|l|}{ ASA score } \\
\hline$\ll 3$ & 1 & - & - & 1 & - & - \\
\hline$\geq 3$ & 2.19 & $1.96-2.43$ & $<0.001$ & 1.42 & $1.22-1.65$ & $<0.001$ \\
\hline \multicolumn{7}{|l|}{ Wound class } \\
\hline 1 & 1 & - & - & 1 & - & - \\
\hline II & 1.74 & $1.54-1.96$ & $<0.001$ & 1.36 & $1.08-1.72$ & $<0.05$ \\
\hline III & 4.11 & $3.55-4.75$ & $<0.001$ & 1.71 & $1.29-2.26$ & $<0.001$ \\
\hline IV & 6.81 & $5.61-8.21$ & $<0.001$ & 2.51 & $1.83-3.44$ & $<0.001$ \\
\hline \multicolumn{7}{|l|}{ Technique of operation } \\
\hline Classic & 1 & - & - & 1 & - & - \\
\hline Videoscopic & 0.57 & $0.49-0.66$ & $<0.001$ & 0.49 & $0.40-0.61$ & $<0.001$ \\
\hline \multicolumn{7}{|c|}{ Hospital stay before operation } \\
\hline$<2$ days & 1 & - & - & 1 & - & - \\
\hline$\geq 2$ days & 2.14 & $1.92-2.39$ & $<0.001$ & 1.22 & $1.05-1.41$ & $<0.05$ \\
\hline \multicolumn{7}{|l|}{ Sex } \\
\hline Male & 1 & - & - & 1 & - & - \\
\hline Female & 0.72 & $0.65-0.80$ & $<0.001$ & 1.10 & $0.96-1.27$ & 0.166 \\
\hline \multicolumn{7}{|l|}{ Age } \\
\hline$<65$ years & 1 & - & - & 1 & - & - \\
\hline$\geq 65$ years & 1.70 & $1.54-1.87$ & $<0.001$ & 1.01 & $0.88-1.16$ & 0.891 \\
\hline \multicolumn{7}{|l|}{ Urgent operation } \\
\hline No & 1 & - & - & 1 & - & - \\
\hline Yes & 1.16 & $1.04-1.29$ & $<0.01$ & 1.29 & $1.11-1.51$ & $<0.05$ \\
\hline \multicolumn{7}{|c|}{ Years of continuous participation in the surveillance } \\
\hline$\ll 2$ years & 1 & - & - & 1 & - & - \\
\hline$\geq 2$ years & 0.60 & $0.53-0.68$ & $<0.001$ & 0.71 & $0.59-0.84$ & $<0.001$ \\
\hline
\end{tabular}

ASA: American Society of Anaesthesiology; Cl: confidence interval; OR: odds ratio.

${ }^{a}$ Multilevel logistic regression. Values reported for fixed effects. Hospitals and operation categories modelled as random effects (both effects significant according to log-likelihood test; p<0.001).

${ }^{b}$ Duration compared with the National Health Surveillance Network 75 th percentile for the given operation category.

the number accounts for about one third of all the infections.

\section{Uni- and multivariate analysis}

Variables commonly associated with higher risk of SSI showed a significantly higher proportion of operations resulting in an infection. Table 2 reports odds ratios (with levels of significance and confidence intervals (CI)) obtained both with univariate and multivariate analysis: longer intervention duration, ASA score of at least three, and duration of pre-surgery hospital stay of at least two days, were found to be associated with an increased risk of SSI, whereas videoscopic procedures were associated with reduced SSI rates.
Operations performed in hospitals with at least two years of surveillance behind them showed a $29 \%$ lower risk of SSI, after accounting for all the other predictors, including the operation category and the facility. When the same model was applied to the severe infections detected in hospital, either before discharge or on readmission, $(n=313 ; 0.5 \%)$, the values obtained for odds ratios and $\mathrm{Cls}$ were similar to the ones from the model on the complete dataset, thus including infections detected in both in- and outpatients. In particular the odds ratio for operations performed in hospitals with at least two years of surveillance was $0.58(95 \%$ $\mathrm{Cl}: 0.36-0.92)$. 


\section{Post-discharge surveillance}

Ten days after the operation, when $90 \%$ of patients were already discharged, barely over a half of the recorded SSIs had been detected. Over $80 \%$ of SSIs were detected by the day 16, and over $90 \%$ by day 22 . The median length of post-intervention follow-up was 26 days (IQR 9-30 days). This figure is very close to the desired complete follow-up of 30 days. The date of last information for surveyed procedures, corresponding to the end of the follow-up, was defined in $39 \%$ of cases through an ambulatory visit, in $22 \%$ cases during hospital stay (either before first discharge, or during a readmission), and in the remaining $39 \%$ patients by telephone call or by returning the post-discharge letter.

The proportion of SSI identified through telephone call or pre-stamped letter was $22 \%$. The proportion of nonsuperficial SSI identified in the post-discharge surveillance (PDS) was $11 \%$. Finally, the proportion of SSI identified through the PDS programme varied among different interventions, between 51 and $96 \%$ (see Table 3).

Operations resulting in an SSI lead to an increased post-operation hospital stay. The global median hospital stay for infected patients was five days (IQR: 2-12 days), and was higher for non-superficial SSIs (eight days; IQR: 1-18 days). The median hospital stay was three days (IQR: 1-6 days) in non-infected operated patients.

\section{Discussion}

The first analysis of the Italian SSI surveillance system had two main results: (i) SSIs occurred at a lower rate for operations performed in hospitals that participate regularly to the surveillance, and (ii) the total number of surgical procedures surveyed doubled over the study period. Further interesting information that emerged from this study was the high proportion of SSI, over $60 \%$, identified through PDS. Finally, the study confirmed that most of the risk factors already known to be associated with an increased or reduced risk of SSI were valid also for the Italian population. In fact, longer intervention duration, an ASA score of at least three and pre-surgery hospital stay of at least two days were found to be associated with an increased risk of SSI. On the other hand, videoscopic procedures were associated with reduced SSI rates.

There are several limitations to this study. As every national surveillance system, SNICh has intrinsic limitations, in particular diagnostic criteria, number of enrolled patients, and intensity of surveillance. Although we used the same definition throughout the country, it is possible that the clinical diagnosis varied between hospitals and even between wards of the same facility. This is at least partly related to the fact that SSI diagnostic criteria are not uniform in the medical literature, and are complex and difficult to apply in a consistent way [4]. Despite this, the most common SSI
TABLE 3

Proportion of surgical site infections identified through post-discharge surveillance, Italy, 2009-2011 ( $\mathrm{n}=1,628)$

\begin{tabular}{|l|c|}
\hline Type of intervention & SSI identified with PDS n (\%) \\
\hline Appendectomy & $33 / 51(65 \%)$ \\
\hline Breast surgery & $150 / 156(96 \%)$ \\
\hline Cholecystectomy & $131 / 162(86 \%)$ \\
\hline Colon surgery & $259 / 508(51 \%)$ \\
\hline Caesarean section & $211 / 222(95 \%)$ \\
\hline Rectal surgery & $69 / 126(55 \%)$ \\
\hline
\end{tabular}

PDS: post-discharge surveillance; SSI: surgical site infection.

definitions have similar capacity to predict outcomes influenced by SSI [4]. As no interventions to improve diagnostic capacity have been performed to date, we feel that it is unlikely that intra-centre diagnostic difference had a considerable impact on SSI trends. On the other hand, since no internal validation of the diagnostic criteria has been performed to date, it is possible that some of the differences in SSI rates could be due to inter-centre diagnostic disparities. We feel that, if a problem of misdiagnosis exists, it has probably been similar over the whole study period.

Furthermore, the relatively short duration of the study, three years, should have also restricted the possibility of intra-centre variation. Low representativeness of the surveyed surgical procedures in our national programme represents a second limitation of the study. Not all regional healthcare systems participated in the surveillance programme, and those that did, surveyed very different numbers of interventions.

As a third limitation, it cannot be excluded in the absence of validation studies that the intensity of surveillance changed over the study period, either decreasing or increasing. However, the reduction in SSI incidence we observed was almost the same as reported by the German surveillance programme KISS (Krankenhaus-Infektions-Surveillance-System), and by the Dutch PREZIES (PREventie van ZIEkenhuisinfecties door Surveillance), i.e. $29 \%$ and $31 \%$ respectively $[18,19]$. Furthermore, the observed reduction in SSI was confirmed when considering severe SSI only, i.e. non-superficial SSI diagnosed during hospital stay (OR: 0.58, Cl: 0.36-0.92); they constitute a more stable sample for comparisons because the variations in performing PDS are eliminated $[20,21]$.

Finally, interventions including a prosthetic implant were excluded. This choice was based upon the difference in follow-up that is needed to rule out an infection with prosthetic material, i.e. one year. Although the exclusion of these interventions may limit the 
comparability with other systems, the proportion of such procedures varies significantly in the different systems, representing from less than a third to over half of the surveyed interventions $[9,19,22]$. Despite these differences the reduction observed in the different systems was similar. We therefore hypothesise that the impact on comparability due to exclusion of orthopaedic intervention is small.

The implementation of a national surveillance programme for SSI is a difficult task, particularly in times of crisis, with financial restraints, staff reductions, and decreased investments, including those in new information technology, all factors that impact on management and efficacy of a surveillance programme. In 2006, the CCM funded the implementation of a surveillance programme focussing on SSI and infections in ICU to collect national data and send information to ECDC [12]. As shown here, the Italian national programme had positive effects such as data collection for the European surveillance system, a national standard for SSI surveillance, regional groups on SSI, and a unique surveillance database for SSI.

The most striking achievement of the project, representing the core target of any surveillance program, was the rapid reduction in SSI incidence within three years; hospitals participating for more than two years had a $29 \%$ reduction in SSI rate. It has to be pointed out that the observed reduction was not due to lower baseline SSI rates in the centres surveying for more than two years. In fact, with the specification of the hospitals as random levels in the multilevel analysis, potential differences in baseline SSI rates have been taken into account. The decrease was probably due to not only the implementation of the surveillance programme but also other prevention interventions that are often associated with such programmes. Our observations confirm what has been previously reported in the literature: a significant reduction in hospital-acquired infections is expected within three years from the implementation of the surveillance programme $[5,9,19,22]$. Interestingly, the same decline was observed after implementation of such systems under different conditions and therefore it seems independent of changes in patient characteristics and technological innovation [9]. Data from France and the Netherlands show that further improvement beyond the third year of surveillance is possible, reaching SSI rate reductions of more than $50 \%$ after five to nine years $[5,19]$.

The number of surgical procedures undergoing surveillance doubled in the three-year study period. Similar results have been observed in the European surveillance programme where the number of surgical procedures undergoing surveillance increased 2.8 -fold between 2004 and 2009 [1]. Participation in the national surveillance system increased also in the United States, where the number of procedures surveyed increased from about 550,000 in the 12-year period between 1992 and 2003, with an average of some 45,000 intervention per year, to about 800,000 in the three-year period between 2006 and 2008, i.e. over 260,000 intervention each year, a more than fivefold increase $[23,24]$.

We would welcome a further and steady increase in participation at national level: if the observed reduction were applied to all surgical procedures performed in the country, some 14,000 SSIs per year could be avoided. This could potentially lead to some 25,000 hospital days less per year, prevent individual suffering, and result in significant financial savings. A recent Italian meta-analysis shows that the average cost of one SSI is about EUR 13,000 [25]. Based upon these estimates, the possible savings after three years would range, for the whole country, between EUR 50 million and EUR 175 million. Furthermore, a decrease in SSI would reduce the number of litigations against hospitals, probably representing even larger economic savings.

In contrast to other surveillance systems, SNICh has a high proportion of infection detected during PDS. The internal structure of our surveillance system implies higher SSI rates in centres performing more accurate PDS, and limits the comparability with other systems, such as KISS or PREZIES, and of participating hospitals within SNICh. Programmes with limited or no PDS detect lower SSI rates. Participation to the SNICh programme is voluntary, and each centre may decide which interventions to survey. The centres that perform surveillance and PDS therefore do so willingly. These aspects could limit the generalisability of the results. Aiming at a wider uptake of the programme, there is a need to identify the most efficient surveillance strategy, which could lead to a revision of PDS duration, probably the most resource-intensive part of the SSI surveillance programme. Data from our study show that restricting PDS to three weeks, i.e. to the period when a patient is generally receiving ambulatory care after a surgical procedure, would identify around $90 \%$ of the events.

In conclusion, our data show that national surveillance programmes are beneficial for health, ethical and financial targets. We feel that a progressive expansion of these programmes should be pursued strongly both at a central and local level; mandatory participation could represent an important public health target.

\section{Acknowledgements}

We thank all physicians and nurses of the participating hospitals for collaborating with the SNICh programme. Financial support: Centro per il Controllo e la Prevenzione delle Malattie (CCM).

Conflict of interest

None declared. 


\section{Authors' contributions}

Conception and design: Massimiliano Marchi, Maria Luisa Moro, Angelo Pan, Davide Resi. Collection and assembly of data: Massimiliano Marchi, Mita Parenti, Davide Resi. Analysis and interpretation of data: Massimiliano Marchi, Filomena Morsillo. Drafting the article: Carlo Gagliotti, Massimiliano Marchi, Angelo Pan. Critical revision of the article for important intellectual content: Carlo Gagliotti, Massimiliano Marchi, Maria Luisa Moro, Angelo Pan, Mita Parenti, Davide Resi. Final approval of the article: Carlo Gagliotti, Massimiliano Marchi, Maria Luisa Moro, Angelo Pan, Mita Parenti, Davide Resi.

\section{The members of the SNICh group were:}

Antonella Agodi, Marcello D’ Errico, Luca Fabbri, Francesco Mana, Lorena Martini, Marisa Mastaglia, Roberto Novati, Renato Pizzuti, Anna Poli, Gaetano Privitera, Vincenzo Puro, Peter Josef Santa, Bruno Sarnelli, Carla Zotti.

\section{References}

1. European Centre for Disease Prevention and Control (ECDC) Surveillance of surgical site infection in Europe 20082009. Stockholm: ECDC; 2012. ISBN 978-92-9193-334-1. doi 10.2900/21096. Available from: http://www.ecdc.europa.eu/ en/publications/Publications/120215_SUR_SSI_2008-2009.pdf

2. Council of the European Union. Council recommendation of 9 June 2009 on patient safety, including the prevention and control of healthcare- - associated infections (2009/C151/01). Official Journal of the European Union. 3 Jul 2009. Available from: http://ec.europa.eu/health/patient_safety/docs/ council_2009_en.pdf

3. Decision No $2119 / 98 /$ EC of the European Parliament and of the Council of 24 September 1998 setting up a network for the epidemiological surveillance and control of communicable diseases in the Community. Official Journal of the European Union. 3 Oct 1998. L 268. Available from: http://eurlex.europa. eu/LexUriServ/LexUriServ.do?uri=CELEX:31998D2119:EN:HTML

4. Gibbons C, Bruce J, Carpenter J, Wilson AP, Wilson J, Pearson $A$, et al. Identification of risk factors by systematic review and development of risk-adjusted models for surgical site infection. Health Technol Assess. 2011;15(30):1-156, iii-iv.

5. Rioux C, Grandbastien B, Astagneau P. Impact of a six-year control programme on surgical site infections in France: results of the INCISO surveillance. J Hosp Infect. 2007;66(3):217-23. http://dx.doi.org/10.1016/j.jhin.2007.04.005

6. European Centre for Disease Prevention and Control (ECDC). Surveillance of healthcare-associated infections in Europe, 2007. Stockholm: ECDC; 2012. ISBN 978-92-9193-327-3. doi 10.2900/18553. Available from: http://www.ecdc.europa.eu/ en/publications/publications/120215_sur_hai_2007.pdf

7. Jarvis WR. Benchmarking for prevention: the Centers for Disease Control and Prevention's National Nosocomial Infections Surveillance (NNIS) system experience. Infection. 2003;31(Suppl 2):44-8.

8. Moro ML, Morsillo F, Tangenti M, Mongardi M, Pirazzini MC, Ragni $P$, et al. Rates of surgical-site infection: an international comparison. Infect Control Hosp Epidemiol. 2005;26(5):442-8. http://dx.doi.org/10.1086/502565

9. Gastmeier P, Schwab F, Sohr D, Behnke M, Geffers C. Reproducibility of the surveillance effect to decrease nosocomial infection rates. Infect Control Hosp Epidemiol. 2009;30(10):993-9. http://dx.doi.org/10.1086/605720

10. Centro nazionale per la prevenzione e il controllo delle malattie (CCM). [National Center for the Prevention and Control of Diseases]. Sistema nazionale sorveglianza delle infezioni del sito chirurgico (SNICh) - protocollo. [National surveillance system for surgical site infections (SNICh) - protocol]. Bologna: ASSR Emilia-Romagna; 2011. Italian. Available from: http://assr.regione.emilia-romagna.it/it/servizi/pubblicazioni/ rapporti-documenti/protocollo-sistema-nazionale-disorveglianza-delle-infezioni-del-sito-chirurgico-snichaggiornamento-dicembre-2011

11. Italian database on hospital activity. Rome: Ministry of Health. [Accessed 27 May 2014]. Available from: http://www.salute. gov.it/imgs/C_17_sissezione_2_listaFile_itemName_1_file.xls

12. European Centre for Disease Prevention and Control (ECDC). Surveillance of surgical site infections in European hospitals
- HAISSI protocol. Version 1.02. Stockholm: ECDC; 2012. ISBN 978-92-9193-320-4. doi 10.2900/12819. Available from: http:// www.ecdc.europa.eu/en/publications/publications/120215 ted_ssi_protocol.pdf

13. Culver DH, Horan TC, Gaynes RP, Martone WJ, Jarvis WR, Emori TG, et al. Surgical wound infection rates by wound class, operative procedure, and patient risk index. National Nosocomial Infections Surveillance System. Am J Med. 1991;91(3B):152S-7S

http://dx.doi.org/10.1016/0002-9343(91)90361-Z

14. Edwards JR, Peterson KD, Mu Y, Banerjee S, Allen-Bridson K, Morrell G, et al. National Healthcare Safety Network (NHSN) report: data summary for 2006 through 2008, issued December 2009. Am J Infect Control. 2009;37(10):783-805. http://dx.doi.org/10.1016/j.ajic.2009.10.001

15. R Development Core Team. R: A language and environment for statistical computing. Vienna: R Foundation for Statistical Computing; 2012. ISBN 3-900051-07-0. Available from: http:// www.R-project.org/

16. Bates D, Maechler M, Bolker B. Ime4: Linear mixed-effects models using $S_{4}$. $R$ package version 0.999375-42. Vienna: $R$ Foundation for Statistical Computing; 2011. Available from: http://CRAN.R-project.org/package=lme 4

17. Fay MP. Two-sided exact tests and matching confidence intervals for discrete data. R Journal 2010;2(1):53-8.

18. Gastmeier P, Geffers C, Brandt C, Zuschneid I, Sohr D, Schwab $\mathrm{F}$, et al. Effectiveness of a nationwide nosocomial infection surveillance system for reducing nosocomial infections. J Hosp Infect. 2006;64(1):16-22. http://dx.doi.org/10.1016/j.jhin.2006.04.017

19. Geubbels EL, Nagelkerke NJ, Mintjes-de Groot AJ, Vandenbroucke-Grauls CM, Grobbee DE, de Boer AS. Reduced risk of surgical site infections through surveillance in a network. Int J Qual Health Care. 2006;18(2):127-33. http://dx.doi.org/10.1093/intqhc/mzi103

20. Ming DY, Chen LF, Miller BA, Anderson DJ. The impact of depth of infection and post-discharge surveillance on rate of surgical-site infections in a network of community hospitals. Infect Control Hosp Epidemiol. 2012;33(3):276-82. http://dx.doi.org/10.1086/664053

21. Brandt C, Sohr D, Behnke M, Daschner F, Rüden H, Gastmeier P. Reduction of SSI rates associated with active surveillance; Infect Control Hosp Epidemiol. 2006;27(12):1347-51. http://dx.doi.org/10.1086/509843

22. Astagneau P, L'Heriteau F, Daniel F, Parneix P, Venier AG, Malavaud S, et al. Reducing surgical site infection incidence through a network: results from the French ISO-RAISIN surveillance system. J Hosp Infect. 2009;72(2):127-34. http://dx.doi.org/10.1016/j.jhin.2009.03.005

23. NNIS System. National Nosocomial Infections Surveillance (NNIS) system report, data summary from January 1992 to June 2003, issued August 2003. Am J Infect Control. 2003;31(8):48198.

http://dx.doi.org/10.1016/j.ajic.2003.09.002

24. Edwards JR, Peterson KD, Mu Y, Banerjee S, Allen-Bridson K, Morrell G, et al. National Healthcare Safety Network (NHSN) report: data summary for 2006 through 2008, issued December 2009. Am J Infect Control. 2009;37(10):783-805. http://dx.doi.org/10.1016/j.ajic.2009.10.001

25. Graf K, Ott E, Vonberg RP, Kuehn C, Schilling T, Haverich A, et al. Surgical site infections-economic consequences for the health care system. Langenbecks Arch Surg. 2011;396(4):4539. http://dx.doi.org/10.1007/s00423-011-0772-0 